\title{
Early Cardiac Changes in Radioiodine Treated Young Athyrotic Patients Receiving Thyroid Stimulating Hormone (TSH) Suppressive Levothyroxine
}

\section{(LT4) Replacement}

\author{
${ }^{1}$ Fatima Begum, ${ }^{2}$ Chaudhury Meshkat Ahmed, ${ }^{3}$ Enamul Kabir, ${ }^{1}$ Nurun Nahar, ${ }^{1}$ Sadia Sultana, \\ ${ }^{2}$ Sajal Banerjee, ${ }^{4}$ Shahana Afroz and ${ }^{5}$ Nazma Zaman \\ ${ }^{1}$ National Institute of Nuclear Medicine and Allied Sciences, BAEC, Dhaka, Bangladesh. \\ ${ }^{2}$ Department of Cardiology, Bangabandhu Sheikh Mujib Medical University, Dhaka, Bangladesh. \\ ${ }^{3}$ Department of Pathology, Sir Salimullah Medical College \& Mitford Hospital, Dhaka, Bangladesh. \\ ${ }^{4}$ Bangladesh Atomic Energy Commission, Dhaka, Bangladesh. \\ ${ }^{5}$ Bangladesh University of Engineering and Technology, Dhaka, Bangladesh.
}

Correspondence: Dr. Fatima Begum, Associate Professor, National Institute of Nuclear Medicine \& Allied Sciences (NINMAS), Bangladesh Atomic Energy Commission (BAEC), BSM Medical University

Campus, Block-D, $8^{\text {th }}$ Floor, Shahbagh, Dhaka-1000. E-mail: fkbegum@yahoo.com

\begin{abstract}
Objective: The objective of this study was to evaluate early changes of heart due to thyroid stimulating hormone (TSH) suppressive levothyroxine (LT4) replacement in young athyrotic patients with differentiated thyroid carcinoma (DTC).

Materials and Methods: Thirty young athyrotic patients with DTC, age ranged 20-39 years after radioactive iodine ablation therapy (RAIT), were selected prospectively. These patients had been receiving $200 \mu \mathrm{g} / \mathrm{day} \mathrm{LT}_{4}$ replacement therapy starting from the third day of RAIT. Age, sex, body mass index (BMI), body surface area (BSA) and life style matched 23 healthy volunteers were recruited in the study. Clinical, thyroid hormones levels and Doppler Echocardiographic and Tissue Doppler Imaging (TDI) data were taken at baseline at two months of initiation of LT4 replacement in patients with DTC and in healthy volunteers. Echocardiographic data of patients with DTC were repeated at second visit after 6-12 months of RAIT and both data compared with control.
\end{abstract}

Results: Diastolic parameters evaluated by Doppler Echocardiography and TDI were significantly different in patients with DTC compared to control. Early diastolic tissue velocity $(\mathrm{Em})$ in patients with DTC showed higher value at second visit compared to control (Em, control subjects, $19 \pm 6 \mathrm{~cm} / \mathrm{sec}$, patients at second visit, $23 \pm 4 \mathrm{~cm}$ /sec, $p<0.03$ ). Late diastolic flow (Am) of patients was higher at two visits $(\mathrm{Am}$, at first visit, $15 \pm 5 \mathrm{~cm} / \mathrm{sec}$; at second visit, $19 \pm 6 \mathrm{~cm} / \mathrm{sec})(p<0.004)$ compared to control $(\mathrm{Am}, 9 \pm 1.6 \mathrm{~cm} / \mathrm{sec})$. Modified Tei index was significantly increased (mean $0.48 \pm 0.06$ ) in second visit in patients with DTC compared to control (mean $0.33 \pm 0.04$ ) and at first visit (mean $0.31 \pm 0.05, p<0.001$ ) respectively.

Conclusion: It is concluded that supraphysiologic dose of LT4 has side effects on heart even within short period of replacement. TDI is an important tool to evaluate the early changes in myocardium.
Key words: Differentiated Thyroid Carcinoma (DTC), Levothyroxine (LT4) replacement, Tissue Doppler Imaging (TDI).

\section{INTRODUCTION}

Conventional mode of treatment of patient with differentiated thyroid carcinoma (DTC) is total thyroidectomy followed by radioactive iodine ablation therapy (RAIT). Patients become athyrotic as their thyroid glands have been totally destroyed by above mentioned procedures (1). Thereafter, patients receive life-long levothyroxine (LT4) supplement. LT4 is given in a dose not only to treat hypothyroid state, but in thyroid stimulating hormone (TSH) suppressive dose. Suppression of pituitary secretion of TSH retards the growth and spread of cancer (1-3). LT4 therapy at suppressive doses has certain cardiac disadvantages including increased heart rate, increased incidence of atrial arrhythmias, enhanced left ventricular (LV) systolic function and increased LV mass and deteriorates existing cardiac problems (4-6).

This study was initiated to observe the earliest effects of LT4 in supraphysiologic dose on heart by color Doppler and Tissue Doppler Imaging (TDI) before changes could be detected by clinical examination and conventional 2D echocardiography. 


\section{MATERIALS AND METHODS}

Thirty patients with DTC of low risk group (T1, N0, M0) treated with total thyroidectomy and ablated by radioiodine therapy were recruited randomly in this prospective case control study as experimental group. They were referred to National Institute of Nuclear Medicine and Allied Sciences (NINMAS), Dhaka, Bangabandhu Sheikh Mujib Medical University (BSMMU) campus, Bangladesh Atomic Energy Commission (BAEC) for

radioiodine ablative therapy after total thyroidectomy during the year 2007-2009. All patients were diagnosed as papillary thyroid carcinoma on histological examination. Age, body surface area (BSA) and lifestyle matched 23 healthy adults were taken as control subjects. Informed consent had been taken from control subjects and all patients before participation in the present study. The consent form was prepared in Bangla.

Inclusion criteria of patients in experimental group included patients with DTC with low risk group, who had undergone total thyroidectomy and subsequently received radioiodine ablative therapy, within age range 20-39 years and serum Tg level was below $2 \mathrm{ngm} / \mathrm{L}$. Healthy adults with same age range, euthyroid state with no chronic cardiopulmonary disease, diabetes and deformity were included in volunteer group. Patients who had any sort of cardiac dysfunction, pulmonary disease and patients in high risk of recurrence and already presented with metastasis, with ECG abnormality, or un-interpretable ECG were excluded from the study.

Initially baseline study of cardiac functions such as clinical symptoms and pulse rate, respiratory rate and blood pressure have been recorded and symptom rating scale score (7) were evaluated in all control subjects and patients at two months of radioiodine therapy. Then experimental group has been requested to come after six months to one year of radioiodine treatment. The patients received conventional dose of LT4 at a dose of $200 \mu \mathrm{gm} /$ day starting from third day of radioiodine therapy. Levothyroxine of same reputed brand was taken by each patient in the morning in empty stomach, half an hour before breakfast. It was advised to take drugs separately which hamper absorption of LT4 specially calcium tablet. Patients had been advised to come three monthly for clinical and biochemical follow up for routine checkup. Cardiac functions were assessed at the two months of LT4 supplement and after 6-12 months at the Department of Cardiology, BSMMU.

A complete motion (M) mode and two dimensional (2D), Color Doppler, spectral and Tissue Doppler Echocardiographic evaluation were performed by using an ultrasound imaging system Siemens Acuson CV70 equipped with a 2.8-3.5 $\mathrm{MHz}$ transducer. Echocardiographic studies were performed by a single blinded experienced cardiologist, unaware of study protocol. Echocardiographic recordings were made with subjects in left lateral decubitus position, with the head elevated 20-30 degrees using conventional parasternal and apical views, according to the standardization of the American Society of Echocardiography (8).

Doppler tracings were acquired during quiet respiration with the transducer positioned at, or slightly left of the apical impulse, according to the previously reported method at averaging five consecutive cycles at a paper speed of $50 \mathrm{~mm} / 100$ $\mathrm{mm} / \mathrm{sec}$.

Tissue Doppler imaging of the mitral annular motion was recorded from the apical 4-chamber view. A 2 $\mathrm{mm}$ sample was placed sequentially at the lateral mitral annulus. Early (Em) and late (Am) diastolic annular velocities were measured from TDI images. The ratio of mitral $\mathrm{E}$ to TDI Em was calculated using lateral side (E/Em lat).

The LV Tei index (myocardial performance index), a combined index of systolic and diastolic function, was also calculated using mitral inflow velocity and LV outflow velocity as previously reported. Tei index, defined as the sum of isovolumic contraction (IVCT) and isovolumic relaxation times (IVRT) divided by ejection time (ET), has been proposed to express global left ventricular function [Tei index= (IVCT+IVRT)/ET] (9-10). Modified Tei índex has been calculated obtaining similar parameters from TDI. 
Statistical analysis: The study was designed to compare patients with DTC with age; BMI and BSA matched normal healthy controls at the time of enrollment. Cardiac morphology and function by clinical examination, echocardiography and TDI at different time points were evaluated. Values were expressed as mean \pm SD with $95 \%$ confidence intervals. Statistical analysis was performed by using SPSS, version 13 software packages for windows. One-way ANOVA was used to compare all the clinical, hormones, Doppler Echocardiographic and TDI data between control subjects and patients at first visit and second visit and between two visits. The post hoc covariates were analyzed at Tukey's HSD (honestly significant difference) test.

A probability $(P)$ value less than 0.05 was considered statistically significant.

\section{RESULTS}

Among 30 patients with DTC, 27 were female and three were male. On the other hand, among 23 healthy adults 13 were female and 10 were male. Patients and control subjects had matched physical characteristics in terms of age, height, weight, BSA and BMI $(p>0.05)$. Control subjects showed serum FT3, FT4 and TSH within normal reference ranges [Normal reference values of NINMAS laboratory are FT3:2.8-9.5 p mol/L; FT4:9.5-25.5 p mol/L and TSH: $0.3-5.0 \mathrm{~m} \mathrm{IU/L]}$. All patients who received LT4 on conventional dose (200 $\mu \mathrm{gm} /$ day) showed mildly high FT3 (10.1 $\pm 0.7 \mathrm{pmol} / \mathrm{L})$, FT4 (27.4 \pm 0.94 $\mathrm{p}$ mol/L) and suppressed TSH $(0.09 \pm 0.05 \mathrm{~m} \mathrm{IU/L})$ at second visit. Significant differences $(p<0.001)$ were noted in hormone status between control subjects and patients at second visit.

Symptom Rating Scale (SRS) score for hyperthyroidism was significantly higher in patients compared to healthy controls. In control group, SRS was $1 \pm 1$ and in the patient group SRS $8 \pm 1 \quad(\mathrm{p}<0.05)$ who received LT4 at TSH suppressive therapy. Increased heart rate, palpitations, heat intolerance, anxiety tremor and nervousness were more evident features among patients and these symptoms are similar to those present in mild hyperthyroid state. Systolic blood pressure (SBP) and diastolic blood pressure (DBP) did not differ in the control subjects and in patients group in subsequent two visits $(p>0.05)$.

In control subjects and patients at baseline study, Echocardiographic data obtained from left ventricular $M$ mode measurements such as interventricular septal thickness (IVST), posterior wall thickness (PWT), left ventricular internal diameter during diastole (LVIDD) and left ventricular internal diameter during systole (LVIDS) and ejection fraction $(\mathrm{EF} \%)$ were comparable $(p>0.05)$ (Table 1). Left ventricular mass (LVM), left ventricular mass index (LVMI), fraction shortening (FS \%) and heart rate adjusted mean velocity of circumferential fiber shortening ( $\mathrm{mVCFc}$ cir/sec) were different in control subjects and patients at two visits (Table 1)

Table 1: Echocardiographic data of left ventricle in control subjects and patient group at first and second visits.

\begin{tabular}{|c|c|c|c|}
\hline Parameters & $\begin{array}{l}\text { Control subjects } \\
\quad(\mathbf{n}=\mathbf{2 3}) \\
\text { mean } \pm \text { SD }\end{array}$ & $\begin{array}{l}\text { Patients at first } \\
\text { visit }(n=30) \\
\text { mean } \pm \text { SD }\end{array}$ & $\begin{array}{c}\text { Patients at second } \\
\text { visit }(\mathrm{n}=30) \\
\text { mean } \pm \text { SD }\end{array}$ \\
\hline IVST (mm) & $8 \pm 1$ & $7 \pm 1$ & $7 \pm 1$ \\
\hline PWT (mm) & $8 \pm 1$ & $7 \pm 1$ & $8 \pm 1$ \\
\hline LVIDD (mm) & $43 \pm 3$ & $44 \pm 3$ & $45 \pm 3$ \\
\hline LVIDS (mm) & $28 \pm 2$ & $28 \pm 2$ & $28 \pm 2$ \\
\hline $\mathrm{EF}(\%)$ & $66 \pm 4$ & $66 \pm 4$ & $66 \pm 3$ \\
\hline LVM (gm) & $103 \pm 21$ & $99 \pm 17$ & $110 \pm 15+t$ \\
\hline LVMi $\left(\mathrm{gm} / \mathrm{m}^{2}\right)$ & $68 \pm 12$ & $66 \pm 10$ & $74 \pm 10 \dagger+$ \\
\hline FS (\%) & $34 \pm 3$ & $35 \pm 3$ & $39 \pm 3+t$ \\
\hline $\mathrm{mVCFc}(\mathrm{cir} / \mathrm{sec})$ & $1.12 \pm 0.12$ & $1.23 \pm 0.14$ & $1.42 \pm 0.18+\frac{1}{t}$ \\
\hline $\begin{array}{l}\text { Peak Aortic } \\
\text { Velocity }(\mathrm{cm} / \mathrm{sec})\end{array}$ & $89 \pm 9$ & $99 \pm 16$ & $102 \pm 13 \dagger t$ \\
\hline ET (ms) & $300 \pm 18$ & $286 \pm 19$ & $274 \pm 26 \dagger+$ \\
\hline \multicolumn{4}{|c|}{$\begin{array}{l}p>0.05 \text { between Control and patient group at first visit. } \\
\dagger p<0.05 \text { Group-II between at first and second visits. } \\
+p<0.05 \text { between control and Group-II at second visit }\end{array}$} \\
\hline
\end{tabular}

All diastolic parameters measured by Doppler Echocardiography are shown in Table 2. Early diastolic flow velocity (E) through mitral valve in both groups (control subjects, $75 \pm 8 \mathrm{~cm} / \mathrm{sec}$, patients at first visit, $79 \pm 12 \mathrm{~cm} / \mathrm{sec}, p>0.05$ ) were observed and found similar. Decreased E value, $73 \pm 9 \mathrm{~cm} / \mathrm{sec}$ was found at second visit compared to first visit value of patients $(p<0.05)$.

Late diastolic flow velocity (A) through mitral valve was increased in patient groups and it was statistically significant higher than control subjects 
(A, control subjects, $44 \pm 5 \mathrm{~cm} / \mathrm{sec}$; patients group, at first visit, $56 \pm 9 \mathrm{~cm} / \mathrm{sec}$, at second visit, $58 \pm 8 \mathrm{~cm}$ $/$ sec, $p<0.05)$. E/A ratio was significantly $(p<0.05)$ decreased in patients at two visits compared to control subjects (Table 2). Decreased deceleration time is noted in patients compared to control subjects (Dct, control, $173 \pm 19 \mathrm{~m} \mathrm{sec}$, patients, at first visit, $150 \pm 20 \mathrm{~m} \mathrm{sec}$, at second visit, $127 \pm 18 \mathrm{~m} \mathrm{sec}, p<0.05$ ) [Table 2]. Tei index which is considered recently the parameter to measure the systolic and diastolic performances of heart. It was measured in control subjects and patients at different visits showing no significant difference (Tei index; control subjects, $0.32 \pm 0.05$; patients at first visit, $0.33 \pm 0.1$, at second visit, $0.31 \pm 0.17, P>0.05$ ) (Table 2 ).

Tissue Doppler imaging at lateral wall of mitral annulus showed that early diastolic peak (Em)in patients showed higher Em level at second visit compared to control subjects (Em, control subjects, $19 \pm 6 \mathrm{~cm} / \mathrm{sec}$, patients at second visit, $23 \pm 4 \mathrm{~cm} / \mathrm{sec}$, $p<0.03$ ) (Table 2). Late diastolic flow (Am) (mean $19 \pm 6 \mathrm{~cm} / \mathrm{sec}$ ) of patients was higher at two visits $(p<0.004)$ compared to control subjects. In few patients, late tissue diastolic flow (Am) were higher or equal to early tissue diastolic flow (Em), showing diastolic abnormality may be due to hyperthyroid state [Figure 2]. In these cases, TDI provided information about early changes of diastolic function. $\mathrm{E} / \mathrm{Em}$ was low at second visit of patients compared to control subjects (Table 2).

Table 2. Diastolic function parameters evaluated by Doppler Echocardiography and Tissue Doppler Imaging in control subjects and patients at first and second visits

\begin{tabular}{|c|c|c|c|}
\hline Parameters & $\begin{array}{c}\text { Control }(n=23) \\
\text { mean } \pm \text { SD }\end{array}$ & $\begin{array}{c}\text { Patients at first } \\
\text { visit }(\mathrm{n}=\mathbf{3 0}) \\
\text { mean } \pm \text { SD }\end{array}$ & $\begin{array}{c}\text { Patients at second } \\
\text { visit }(n=30) \\
\text { mean } \pm \text { SD }\end{array}$ \\
\hline $\mathrm{E}(\mathrm{cm} / \mathrm{s})$ & $75 \pm 8$ & $79 \pm 12$ & $73 \pm 9 \dagger$ \\
\hline $\mathrm{A}(\mathrm{cm} / \mathrm{s})$ & $44 \pm 5$ & $56 \pm 9$ & $58 \pm 8 \div \dagger$ \\
\hline $\mathrm{E} / \mathrm{A}$ & $1.7 \pm 0.3$ & $1.4 \pm 0.2$ & $1.29 \pm 0.2 \pm \dagger$ \\
\hline IVRT (ms) & $84 \pm 6$ & $76 \pm 9$ & $71 \pm 8+\dagger$ \\
\hline Dct (ms) & $173 \pm 19$ & $150 \pm 20$ & $127 \pm 18 \div \dagger$ \\
\hline Tei index & $0.32 \pm 0.05$ & $0.33 \pm 0.1$ & $0.31 \pm 0.17$ \\
\hline \multicolumn{4}{|c|}{ Lateral mitral annular } \\
\hline $\operatorname{Em}(\mathrm{cm} / \mathrm{s})$ & $19 \pm 3$ & $22 \pm 3$ & $23 \pm 4 \dagger$ \\
\hline $\mathrm{Am}(\mathrm{cm} / \mathrm{s})$ & $9 \pm 1.6$ & $15 \pm 5$ & $19 \pm 6+t$ \\
\hline $\mathrm{E} / \mathrm{Em}$ & $4 \pm 0.8$ & $3.5 \pm 1$ & $3 \pm 1 \ddagger$ \\
\hline Mod Tei & $0.33 \pm 0.04$ & $0.31 \pm 0.05$ & $0.48 \pm 0.06+t$ \\
\hline \multicolumn{4}{|c|}{$\begin{array}{l}p>0.05 \text { between Control and patient group at first visit. } \\
\dagger p<0.05 \text { Group-II between at first and second visits. } \\
\ddagger p<0.05 \text { between control and Group-II at second visit }\end{array}$} \\
\hline
\end{tabular}

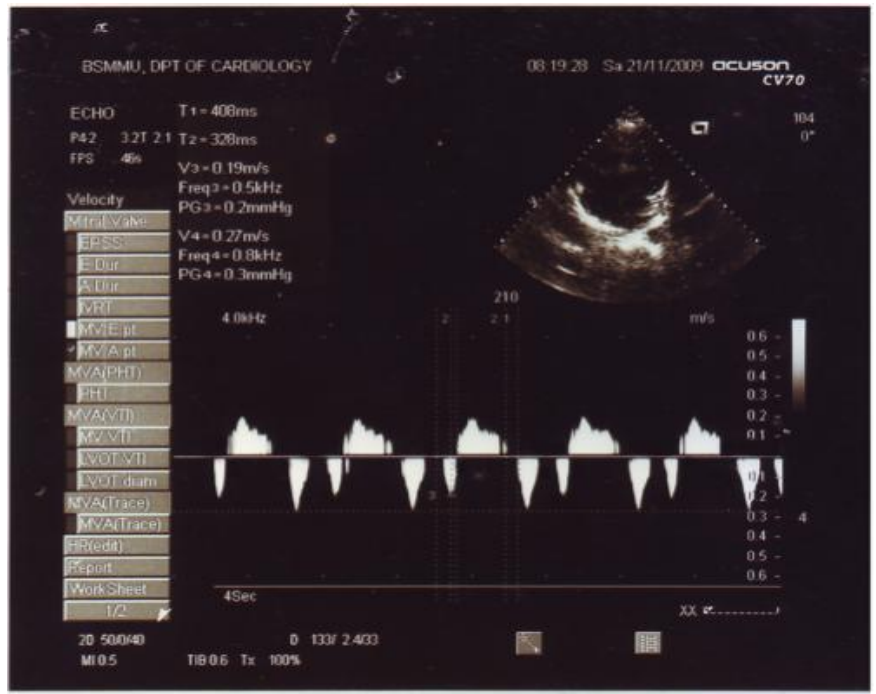

Figure 1: Normal pattern of $\mathrm{Em}(27 \mathrm{~cm} / \mathrm{sec})$ and $\mathrm{Am}$ $(21 \mathrm{~cm} / \mathrm{sec})$ waves of tissue Doppler imaging of a patient with differentiated thyroid carcinoma on LT4 therapy showing slightly high velocities.
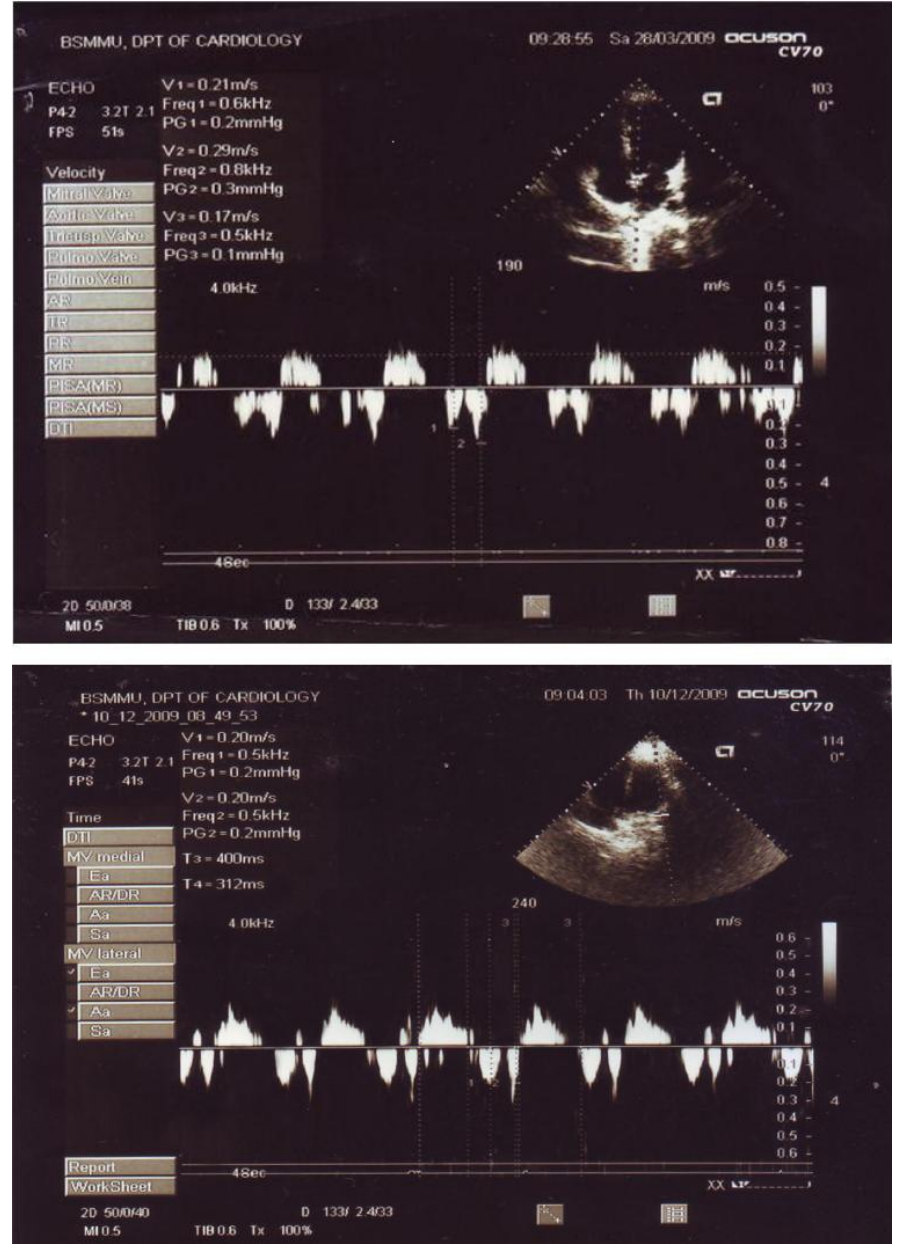

Figure 2: Abnormal pattern (reverse) of Em and Am waves observed in two different patients of group-II at second visit indicating diastolic abnormality. 
Significantly increased modified Tei index was observed (mean $0.48 \pm 0.06$ ) in last follow up of patients compared to value of Mod Tei index of control subjects (mean $0.33 \pm 0.04$ ) and patients at first visit (mean $0.31 \pm 0.05)(\mathrm{p}<0.001)$. Similar Tei Index was observed in the patients at all-time points (Table 2) in Doppler echocardiography.

\section{DISCUSSION}

Patients with DTC receive lifelong LT4 therapy after total thyroidectomy and radioiodine ablative therapy. LT4 is given at TSH suppressive dose to keep the patients in subclinical hyperthyroid state to prevent metastases lowering the stimulus for follicular growth by this hormone. Subsequent follow up strategies vary according to the patient's individual risk (3). Different institutes follow their own treatment schedule or protocol for LT4 replacement. At our institute, usual practice of LT4 replacement dose is $200 \mu \mathrm{gm} /$ day/patient, commencing on $3^{\text {rd }}$ day of therapy for initial 3 months. Thereafter, LT4 dose is adjusted with the hormone levels (FT3, FT4 and $\mathrm{TSH}$ ) to keep the levels in TSH suppressive condition. The purpose of the present study was to detect the early side effects of supraphysiologic dose of LT4 on heart by Color Doppler Echocardiography and TDI in young athyrotic patients with DTC.

In this present study, female to male ratio of patients with DTC was 9:1, which has reflected the incidence sex pattern of DTC. Similar sex patterns of patients with DTC had been observed in some previous studies $(5,11)$. All patients were given LT4 at dose of $200 \mu \mathrm{gm} /$ day, commencing on $3^{\text {rd }}$ day of the radioiodine ablative therapy. This replacement therapy of LT4 has been being practiced in our country and also in some institutes of neighboring country, India (12).

In patients with DTC with adequate TSH suppression may assure long survival (1-3). On the other hand, patients with DTC who are usually kept in TSH suppressed state suffer from some symptoms and signs of thyrotoxicosis $(4,11)$. Although, American Thyroid Association (3) advocated that patients with high risk DTC are to be maintained in suppressed TSH level condition but patients with low risk group DTC are to be kept in euthyroid state with low normal TSH level. In the present study, echocardiographic data obtained from left ventricular $M$ mode measurements such as IVST, PWT, LVIDD and LVIDS were comparable in control subjects and patients at baseline study (Table 1). This may be due to short period of observation. Cardiac anatomical changes such as increment of wall thickness were found after prolonged exposure of LT4 at suppressive dose in other studies $(5,11)$.

All diastolic parameters measured by Doppler Echocardiography are shown in Table 2. Early diastolic flow velocity (E) through mitral valve in both groups were observed and found similar. Decreased $E$ value was found at second visit compared to first visit value of patients. Late diastolic flow velocity (A) through mitral valve was increased in patients and it was statistically significant higher than control subjects (Table 2). Diastolic dysfunction may occur in hyperthyroid state. E wave and late diastolic flow velocity, A wave of control subjects of present study were observed similar with the normal range of Japanese population found in the study by Daimon $\mathrm{S}$ et al., 2008 (13).

E/A ratio was significantly $(p<0.05)$ decreased in patients at two visits compared to control subjects [Table 2]. Decreased deceleration time is noted in patients compared to control subjects [Table 2]. This decreased deceleration time is due to hyperthyroid state in patients on LT4 replacement causing hypercontractility of heart. It has been mentioned in previous literatures that thyroid hormone cause decrement of $\mathrm{E}$ wave due to rapid heart rate and decreased ventricular filling due to low distensibility (11).

TDI at lateral wall of mitral annulus showed that early diastolic peak (Em) in patients showed higher Em level at second visit compared to control subjects $(p<0.03)$ [Table 2]. Late diastolic flow (Am) (mean $19 \pm 6 \mathrm{~cm} / \mathrm{sec}$ ) of patients was higher at two visits $(p<0.004)$ compared to control subjects. In few patients, late tissue diastolic flow was higher or equal to early tissue diastolic flow, showing diastolic abnormality may be due to hyperthyroid state [Figure 2]. As a result mean Am velocity was increased. In some of the above mentioned cases 
Doppler E and A waves were normal but reverse pattern is seen in Em and Am which indicating 'pseudo normalization' of diastolic abnormality (14). In these cases, TDI provides information about early changes of diastolic dysfunction. E/Em was low at second visit of patients compared to control subjects (Table 2).

Significantly increased modified Tei index was observed in last follow up of patients compared to value of Mod Tei index of control subjects and patients at first visit $(p<0.001)$ [Table 2]. On the other hand, similar Tei Index was observed in the patients at all time points. This parameter depends on isovolumic relaxation time (IVRT) (Table 2) and this relaxation time is decreased due to hypercontractility of heart influenced by LT4 therapy (5). Tei index is a conceptually new, simple and reproducible Doppler index of combined systolic and diastolic myocardial performance in patients with primary myocardial systolic dysfunction. Tei index is dependent on heart rate, preload and after load, but Modified Tei index is not dependent on those. Another advantage of modified Tei index, it is measured by the parameters (IVRT, ET) in same cardiac cycle (9-10).

LT4 replacement in supraphysiologic doses within short period of time may cause early changes in heart before advanced pathological changes as mentioned above. These changes could be assessed by TDI before evident by 2D echocardiography. These findings may be explained by hypercontractility of heart due to overexposure to LT4. Long-term administration of LT4 in TSH suppressive dose has some ill effects on heart such as increased heart rate, atrial fibrillation, left ventricular hypertrophy, systolic and diastolic dysfunction $(5,12,13)$. In several studies, it has been shown that chronic replacement therapy with suppressive doses of LT4 impairs quality of life and psychometric functionality in patients DTC $(4,5,14)$. Thereby, optimization of dose of LT4 may be considered in young low risk DTC patients to preserve good cardiac function and quality of life.

Limitation of this study was small study population. Further studies are recommended in larger scale with more consecutive observations by TDI.

\section{CONCLUSION}

Chronic exposure of mild excess of LT4 replacement causes untoward effects on heart. Those early side effects can be elicited by Color Doppler echocardiography and TDI before these changes are evident in clinical or 2D Echocardiography findings. Thereby, physician can be cautious about dosing of LT4 especially in young athyrotic patients with low risk group DTC to preserve good cardiac function and maintain quality of life.

\section{Disclosure of conflict of interest:}

The authors declare that there is no conflict of interests regarding the publication of this paper.

Acknowledgments: This article is the partial work of $\mathrm{Ph} \mathrm{D}$ thesis of first author obtained from Bangladesh University of Engineering and Technology (BUET), 2010. This research work was approved and funded by Committee for Advanced Studies and Research (CASR) of BUET, No:

DAERS/CASR/R-01/2008/D-1321/114.We are sincerely grateful to authority of BUET for funding the study and Department of Cardiology, Bangabandhu Sheikh Mujib Medical University, Dhaka for cooperation.

\section{REFERENCES}

1. Schlumberger M, Berg G, Cohen O. Follow-up of low-risk patients with differentiated thyroid carcinoma: a European perspective. European $\mathbf{J}$ Endocrinol 2004; 150:105-12.

2. Pujol P, Daures JP, Nsakala N, Baldet L, Bringer J and Jaffiol C. Degree of Thyrotropin Suppression as a Prognostic Determinant in Differentiated Thyroid Cancer. J Clin Endocrinol Metab1996;81:4318-23.

3. Cooper DS, Deherty GM, Haugen BR, Kloos RT, Lee SL, Mandel SJ, et al. RevisedAmerican Thyroid Association Management Guidelines for Patients with Thyroid Nodules and Differentiated Thyroid Carcinoma. Thyroid 2009; 19(11): 1167-214.

4. Biondi B, Fazio S, Carela C, Amato G, Cittadini G, Lupoli $\mathrm{G}$ et al. Cardiac Effects of Long Term

Thyrotropin Suppressive Therapy with Levothyroxine. J Clin Endocrinol Metab1993; 77(2): 334-38. 
5. Biondi B, Palmieri EA, Fazio S, Cosco S, Nocera M, Sacca Let al. Endogenous Subclinical Hyperthyroidism Affects Quality of life and Cardiac Morphology and Function in Young and Middle-aged Patients. J Clin Endocrinol Metab 2000; 85:4701-05.

6. Klein I and Danzi S. Thyroid disease and the heart. Circulation 2007; 116:1725-35.

7. Klein I, Trzepacz PT, Roberts M, Levey GS. Symptom Rating Scale for Assessing Hyperthyroidism. Arch Intern Med 1988; 48:387-90.

8. ACC/AHA guidelines for the Clinical Applications of Echocardiography Executive Summary. A report of the American College of Cardiology/ American Heart Associations Task Force on Practice Guidelines (Committee on Clinical Application of Echocardiography. J Am Coll Cardiol 1997; 29:86279.

9. Keser N, Yildiz S, Kurtoglu N, Dinder I. Modified Tei Index: A promising Parameter in Essential Hypertension. Echocardiography 2005; 22(4):296304.
10. Karatzis EN, Giannakopoulou AT, Papadakis J E, Karazakos AV, Nearchou NS. Myocardial Performance Index (Tei index). Evaluating its Application to Myocardial Infarction. Hellenic J Cardiol 2009; 50:60-65.

11. Biondi B, Fazio S, CuocoloA, Sabatini D, Nicolai E, Lombardi $G$ et al. Impaired Cardiac Reserve and Exercise Capacity in Patients Receiving Long-Term Thyrotropin Suppressive Therapy with Levothyroxine. J Clin Endocrinol Metab 1996;81: 4224-28.

12. Samuel AM and Rajashekhar RB. Radioiodine Therapy For Differentiated Thyroid Cancer. In: Rao R S, Samuel AM, and Shah DH. Thyroid Cancer An Indian Perspective. Mumbai, Quest publications. 1999:213-73.

13. Daimon M, Watanabe $H$, Abe $Y$, Hirata $K$, Hozumi T,Ishii K. Normal Values of Echocardiographic Parameters in Relation to Age in a Healthy Japanese Population - The JAMP Study. Circ J 2008;72:185966.

14. Mercuro G, Panzuto MG, Bina A, Leo M, Cubola S and Petrini L. Cardiac Function, Physical Exercise Capacity, and Quality of life during Long- term Thyrotropin-Suppressive Therapy with Levothyroxine: Effect of Individual Dose Tailoring. J Clin Endocrinol Metab 2000; 85:159-64. 